\title{
Review \\ Towards a global entrepreneurial culture: a systematic review of the effectiveness of entrepreneurship education programs
}

\author{
Jacinto Jardim", Ana Bártolo², Andreia Pinho ${ }^{3 *}$
}

1 Entrepreneurship Education Office, Department of Social Sciences and Management, Universidade Aberta, 1250-100 Lisboa, Portugal; jacinto.jardim@uab.pt (J.J.)

2 Center for Health Technology and Services Research (CINTESIS), Department of Education and Psychology, University of Aveiro, 3810-193 Aveiro, Portugal; anabartolo@ua.pt (A.B.)

3 Entrepreneurship Education Office, Universidade Aberta, 1250-100 Lisboa, Portugal; andreia.pinho@uab.pt (A.P.)

* Correspondence: jacinto.jardim@uab.pt; Tel.: +351-968-069-643

\begin{abstract}
The number of entrepreneurship education programs (EEP) has increased exponentially over the past two decades. However, a systematic review has not yet been carried out to confirm the effectiveness of EEPs and their presence in the current global world. The main objective of this study was to provide a systematic synthesis of EEP, exploring their characteristics and effectiveness. The search was carried out in the following databases: Scopus, Web of Science, ProQuest and ERIC. Twenty-nine articles were included, with programs developed mainly in European $(n=15)$, Asian $(n=6)$ and American $(n=5)$ countries. The programs were mainly aimed at higher education students $(n=17)$, addressing the development of business plans and entrepreneurial skills. However, greater attention is paid to the entrepreneurial skills in both basic and secondary education. The development of the programs under analysis varied between one week and two years. The results of the studies showed the effectiveness of most of these programs in promoting entrepreneurial skills at all levels of education. In turn, there was not a verified increase in the intention to start a business, since this intention is determined by predisposition, namely socio-cultural and family aspects. This systematic review of the EEP points to the need for this type of program to be preferentially developed since the early school years, since it is at that time that predispositions are created for the development of entrepreneurial skills and intentions. This condition is corroborated by the global geography of the EEP, which demonstrates that, where there is currently an entrepreneurial culture, countries have made a long educational journey, with strategic options from the perspective of educational policies defending entrepreneurship among the younger generations.
\end{abstract}

Keywords: entrepreneurial programs; pedagogical interventions; educational interventions; entrepreneurship education; entrepreneurial culture; effectiveness; globalization 


\section{Introduction}

Education towards entrepreneurship (EE) is, par excellence, a field for the advancement and development of societies, a trigger for economic growth, social cohesion, organisational success and personal fulfilment [1-5]. For this reason, over the past two decades, entrepreneurship education programs (EEP) have increased exponentially on all continents [6-15]. This educational phenomenon originated at Harvard Business School, where the first entrepreneurship course, called Management of New Enterprises, was offered by Myles Mace. A year later, a research centre in this field was created, the Research Centre in Entrepreneurial History [16,17]. However, courses began to appear at various universities in the USA, and in 1967 the first MBAs on entrepreneurship were held at Stanford University and New York University [18]. In the following year, 1968, Babson College offered the first degree in entrepreneurship [16].

This phenomenon has been spreading all over the world, given its relevance in the solution of emerging problems. However, if initially the focus was on the creation and management of companies, in recent decades the focus has also opened up to entrepreneurial skills, attitudes and behaviours [1,19-21]. Education towards entrepreneurship began to take an approach that makes it possible to cover students from all higher education courses, as well as all levels of education, who need to develop these skills. Therefore, entrepreneurial programs have been developed and implemented on all continents, as a way of preparing and enabling them to face professional challenges, to create their own jobs, to create original and valuable solutions to various emerging social and economic problems, such as the environment, poverty, social exclusion and sustainability. And the EE assumed the objective of promoting an entrepreneurial culture, having the aforementioned competences as a frame of reference [22-24].

With this objective, interest in educational actions in the form of intervention programs has grown, consisting in intentional and systematic actions, resulting from the identification of the needs of a population, directed to specific objectives, based on theoretical models, and suggesting activities and pedagogical resources for their execution and evaluation, as defined by Jardim [25]. Consequently, studies on these interventions have been carried out on all continents, with the most diverse recipients, such as students in higher education [26,27], primary school [28-32], secondary education [33-35]; as well as the most diverse contexts outside the school, such as municipal [36,37], agricultural [38], and retirement [42]. These references show the fact that the actions of education towards entrepreneurship have multiplied around the world in recent years, which demonstrates the need for entrepreneurial skills. A paradigmatic example is that of EEP in Hong Kong secondary schools, presented by Cheung [9], who concluded that $70 \%$ of schools apply EEP for more than three years; that in most programs (48\%) the activities are carried out over one or more school years; that several teaching approaches are adopted, predominantly workshops (46\%), competitions, case studies, and mentoring. However, $75 \%$ of schools also use traditional teaching methods.

Thus, the good results achieved through the EEP come from the programs' conceptions, namely the leveled objectives, the competences, the connection with the socio-economic context, the profile of the program facilitator, the activities carried out, as has been demonstrated in the literature [10,15,29,40-43]. Thus, according to Jardim [25], there are three dimensions to be taken into account when designing an intervention program: identity of the program (name of the program, characterization of the recipients and their socio-educational context, objectives, content and skills, theoretical foundation); pedagogical options (activities, resources, number of sessions, total duration, facilitators); and evaluation (research design, sample definition, assessment tools, results and implications for subsequent interventions).

One of the constituent elements of the EEP is entrepreneurial skills (ESs), which enable students to face the challenges of the current labour market, such as the sense of initiative, problem-solving, innovation, creativity and teamwork. For this reason, several 
approaches and models of ESs have emerged [44-52]. These skills have been progressively included in school curricula and interventions have been carried out to promote them at all ages. Thus, it is possible to disseminate an entrepreneurial culture for all, so that the economy and the market include not only those who were born in a family and socioeconomic context favourable to entrepreneurship, but also those who, through learning and training, acquire the skills, competences, values, emotions, and tools of this culture [1].

We assume that the development of the elements of entrepreneurial culture requires the creation of an educational environment favourable to the creation of value proposals, in original projects, products, or services from the point of view of innovation, useful for certain recipients and financially viable. This teaching-learning process implies the use of a set of specific pedagogical strategies. They can be educational games, biographies of entrepreneurs, group dynamics or business models, and can be in digital or printed format, virtual or in-person, individual or in the group. As an example, we can observe some of these teaching-learning processes in Europe [53,54] and in the world [14,55]. And there is a wide variety of procedures and resources, which represent evidence of the diversity of proposals according to economic, cultural and social contexts [1]. Considering this multiplicity of approaches, a model composed of twelve strategies distributed over four objective domains was presented: tools to develop ideas, which are intended to be original, useful and profitable; tools for the validation and dissemination of projects; tools to communicate effectively and quickly with customers; and tools to provide sustainability to organisations.

Thus, there was a clear need to design and implement EEP, aiming at promoting entrepreneurial skills, to disseminate an entrepreneurial culture in current society that requires creativity and innovation from all professionals in solving emerging problems. And multiple studies on entrepreneurship education have been carried out. However, in relation to the EEP, a systematic literature review that describes and evaluates their effectiveness has not yet been carried out. For this reason, the main objective of this study was to conduct an overview of studies that develop EEP and explore its effectiveness in promoting ESs.

\section{Method}

This systematic review was conducted following the Preferred Reporting Items for Systematic Reviews and Meta-Analyses (PRISMA) guidelines [56].

\subsection{Search strategy and eligibility criteria}

A systematic search of the published literature was performed using four databases: Scopus, Web of Science, ProQuest, and ERIC. The keywords used were "entrepreneurial education program*", "pedagogical interventions", "educational interventions", and "entrepreneurship education". The OR and AND functions were also used to combine the key terms. The searches were carried out in January 2021 and were complemented by a manual search of the reference lists of the included studies.

The studies included in this review met the following criteria: they (i) assessed the effectiveness of the EEP and (ii) were published in English, Spanish, and French between 2000-2021. No reviews or meta-analyses, conference abstracts, comments, dissertations, or editorials were included in this study.

\subsection{Process of data extraction and synthesis}


The survey identified potential eligible entries. After removing duplicate records, the titles and abstracts were independently screened by two co-authors. Based on this process, a list of studies for "full-text examination" was produced. Entries that did not meet the inclusion requirements were excluded, namely those that did not identify a program and/or whose focus was not entrepreneurship. All questions were discussed and resolved through discussion with a third reviewer. For each selected study, information was collected within the following categories: research design, sample size, assessment instruments, results, objectives, theoretical model, pedagogical activities and resources, number of sessions, total duration, and facilitator. A narrative synthesis of the studies included in this review was carried out due to the heterogeneity of data related to the design, type of program, measures used to evaluate the results and impact.

\subsection{Critical appraisal}

The included studies were critically assessed by the review team. The assessment was carried out using the Joanna Briggs Institute (JBI) Statistics Assessment and Review Instruments critical appraisal checklists [57-59] for quasi-experimental studies, case reports, case-control studies and qualitative studies. Only articles in which more than 50\% of the JBI criteria were met were included in the review, following the procedure by Bartolo et al. [60]. It should also be noted that the disagreements between the members of the review team in the evaluations were resolved by discussion.

\section{Results}

The study selection process is represented in the flowchart of Figure 1. As indicated, a total of 604 studies were identified through database research and 27 additional records were identified by back citation. After removing 11 duplicate records, 620 studies were analysed based on the title and summary, but 567 did not meet the eligibility criteria. The full text was retrieved for 53 studies, of which 24 were excluded. Among the deleted records, some studies addressed the theme of entrepreneurship but did not describe a program and its evaluation. Thus, 29 studies, published between 2003 and 2020, were included in the review. 


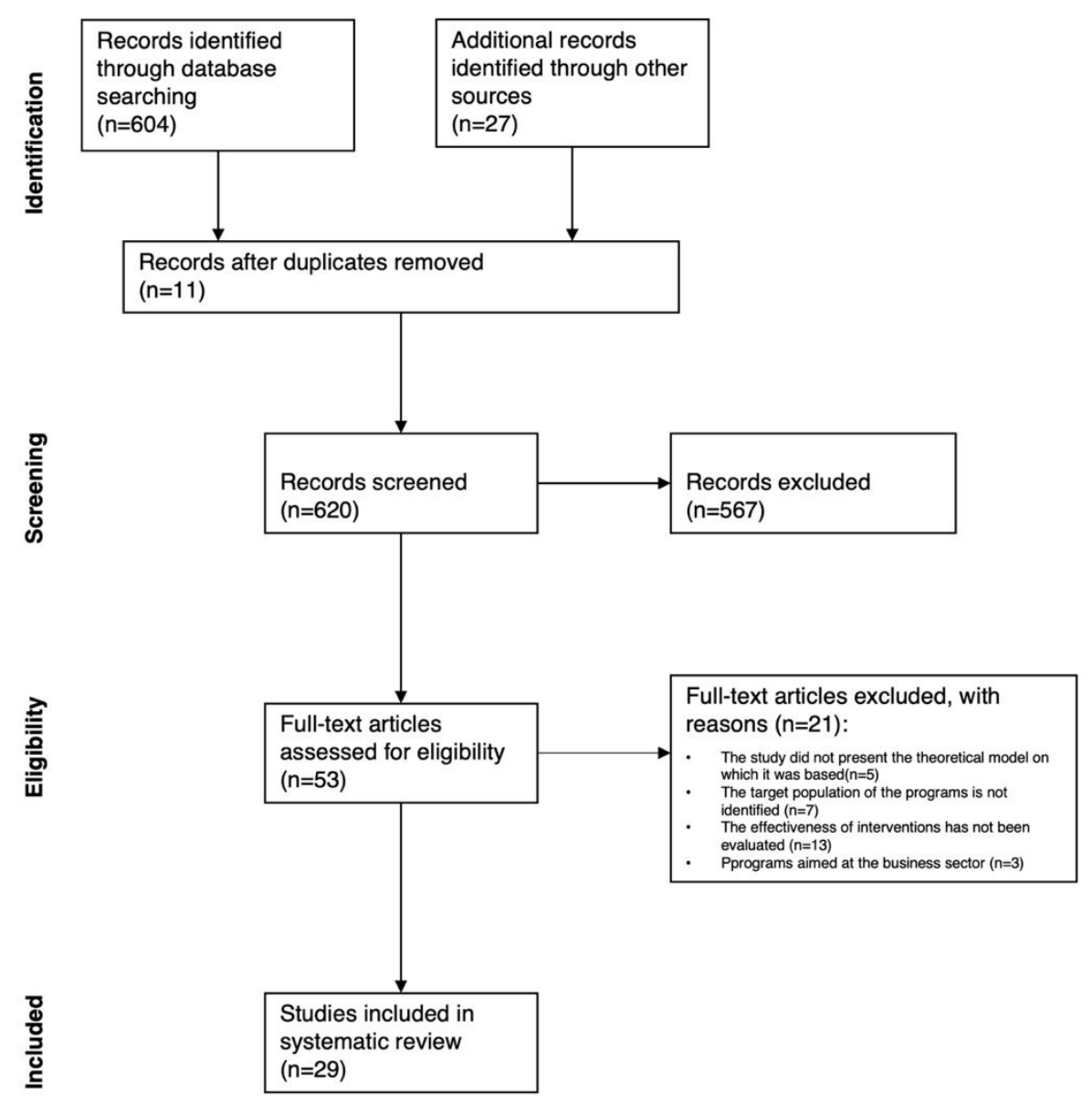

Figure 1. Flowchart with the description of the study selection process for inclusion in the systematic review. Note: Adapted from Moher et al. [61]

\subsection{Characterization of the studies}

The 29 studies included are from six continents and are distributed in 22 countries, three from the USA and two from France, two from South Korea, two from Portugal, two from Spain and two from Taiwan; the remaining studies come from 16 countries, including Germany, Australia, Austria, Botswana, Canada, Chile, Slovenia, Finland, Ghana, the Netherlands, Italy, Malaysia, Norway, the United Kingdom, Romania and Singapore. Most of the programs were envisioned for participants from a single country, however, one of them took place in a partnership between Italy, Germany and Slovenia, as shown in Table 1 - General characterization of the programs.

Thus, this results in a global roadmap of the EEP geography in the current world, where all continents are represented. 
Table 1. General characterisation of the programs

\begin{tabular}{|c|c|c|c|c|c|c|c|c|c|c|c|}
\hline Author, Year & Country & $\begin{array}{l}\text { Sampl } \\
\text { e }\end{array}$ & $\begin{array}{l}\text { Research } \\
\text { design }\end{array}$ & Program name & Recipients & Outcomes & $\begin{array}{l}\text { Sex - } \\
\text { Mal } \\
\text { e }\end{array}$ & $\begin{array}{l}\text { Sex- } \\
\text { Femal } \\
\text { e }\end{array}$ & Ages & $\begin{array}{l}\text { Total } \\
\text { training } \\
\text { hours }\end{array}$ & $\begin{array}{l}\text { Program } \\
\text { facilitator }\end{array}$ \\
\hline $\begin{array}{c}\text { Backs et al., } \\
2019 \text { [62] }\end{array}$ & Germany & 43 & $\begin{array}{l}\text { Qualitative } \\
\text { design }\end{array}$ & $\begin{array}{c}\text { Practice in } \\
\text { Entrepreneurshi } \\
\text { p }\end{array}$ & $\begin{array}{l}\text { Higher } \\
\text { Education }\end{array}$ & $\begin{array}{l}\text { Entrepreneurial } \\
\text { skills }\end{array}$ & 25 & 18 & $18-25$ & $\begin{array}{l}6 \text { months - } \\
36 \text { hours }\end{array}$ & $\begin{array}{l}\text { Teachers and } \\
\text { entrepreneurs }\end{array}$ \\
\hline
\end{tabular}

\begin{tabular}{|c|c|c|c|c|c|c|c|c|c|c|}
\hline $\begin{array}{c}\text { Bernal } \\
\text { Guerrero et al., } \\
2017 \text { [63] }\end{array}$ & Spain & 52 & $\begin{array}{c}\text { Mixed } \\
\text { method } \\
\text { study design }\end{array}$ & $\begin{array}{c}\text { Emprender en } \\
\text { mi Escuela + } \\
\text { Empresa Joven } \\
\text { Europea + } \\
\text { ÍCARO }\end{array}$ & $\begin{array}{l}\text { Primary and } \\
\text { middle } \\
\text { education }\end{array}$ & $\begin{array}{l}\text { Entrepreneurial } \\
\text { skills }\end{array}$ & 26 & 26 & $\begin{array}{l}\text { 10-12, } 9 \text { months - } \\
14-16 \quad 36 \text { hours }\end{array}$ & Teachers \\
\hline
\end{tabular}

\begin{tabular}{|c|c|c|c|c|c|c|c|c|c|}
\hline $\begin{array}{c}\text { Bisanz et al., } \\
2020[64]\end{array}$ & Austria & 139 & $\begin{array}{c}\text { Qualitative } \\
\text { design }\end{array}$ & $\begin{array}{c}\text { Empowering } \\
\text { Each Child }\end{array}$ & $\begin{array}{l}\text { Primary } \\
\text { education }\end{array}$ & $\begin{array}{l}\text { Self-confidence, } \\
\text { spirit of } \\
\text { initiative, } \\
\text { innovation, } \\
\text { creativity, } \\
\text { mindfulness, } \\
\text { empathy, self- } \\
\text { motivation and } \\
\text { participation in } \\
\text { society }\end{array}$ & $25-60$ & $\begin{array}{c}\text { During } \\
\text { the field } \\
\text { trial a } \\
\text { two-year } \\
\text { in-service } \\
\text { training, } \\
\text { consisting } \\
\text { of } 3 \\
\text { training } \\
\text { courses } \\
\text { per year }\end{array}$ & Teachers \\
\hline
\end{tabular}

$\begin{array}{ccccccccc}\begin{array}{c}\text { Boldureanu et } \\ \text { al., 2020 [65] }\end{array} & \text { Romania } & 30 & \begin{array}{c}\text { Mixed } \\ \text { method } \\ \text { study design }\end{array} & \begin{array}{c}\text { Business } \\ \text { Creation }\end{array} & \begin{array}{c}\text { Higher } \\ \text { Education }\end{array} & \begin{array}{c}\text { Entrepreneurial } \\ \text { skills }\end{array} & 21 & 22-46 \text { months - } \\ 36 \text { hours }\end{array}$

\begin{tabular}{|c|c|c|c|c|c|c|c|c|c|c|c|}
\hline $\begin{array}{c}\text { Dominguinho } \\
\text { s \& Carvalho, } \\
2009 \text { [66] }\end{array}$ & Portugal & 22 & Case study & Projeto Começar & $\begin{array}{c}\text { Professional } \\
\text { s }\end{array}$ & $\begin{array}{l}\text { Business and } \\
\text { entrepreneurial } \\
\text { skills }\end{array}$ & 11 & 11 & $25-29$ & $\begin{array}{l}924 \text { hours } \\
\text { (6 months] }\end{array}$ & $\begin{array}{l}\text { Academic } \\
\text { tutor in } \\
\text { higher } \\
\text { education } \\
\text { institution } \\
\text { and business } \\
\text { tutor }\end{array}$ \\
\hline $\begin{array}{c}\text { Fayolle \& } \\
\text { Gailly, 2009 } \\
{[67]}\end{array}$ & France & 158 & $\begin{array}{l}\text { Quasi- } \\
\text { experimenta } \\
\text { l design }\end{array}$ & $\begin{array}{l}\text { Programme } \\
\mathrm{d}^{\prime} \text { Enseignement } \\
\text { en } \\
\text { Entrepreneuriat }\end{array}$ & $\begin{array}{c}\text { Higher } \\
\text { Education }\end{array}$ & $\begin{array}{l}\text { Entrepreneurial } \\
\text { behaviour }\end{array}$ & - & - & $\begin{array}{c}23 \\
\text { more }\end{array}$ & 24 hours & Teachers \\
\hline $\begin{array}{l}\text { Hebles et al., } \\
2019 \text { [68] }\end{array}$ & Chile & 38 & $\begin{array}{c}\text { Qualitative } \\
\text { design }\end{array}$ & $\begin{array}{l}\text { Programa de } \\
\text { Educación en } \\
\text { Emprendimiento } \\
\text { e Innovación }\end{array}$ & $\begin{array}{l}\text { Higher } \\
\text { Education }\end{array}$ & $\begin{array}{c}\text { Entrepreneurial } \\
\text { skills and } \\
\text { behaviour }\end{array}$ & 19 & 19 & $18-25$ & $\begin{array}{l}9 \text { months - } \\
36 \text { hours }\end{array}$ & Teachers \\
\hline
\end{tabular}




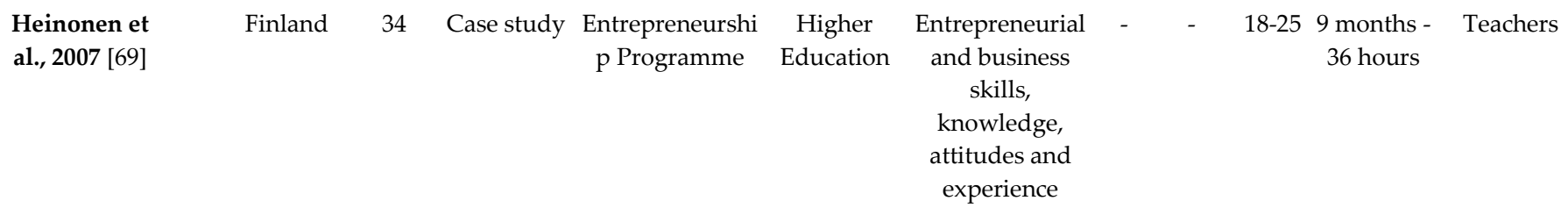

\begin{tabular}{|c|c|c|c|c|c|c|c|c|c|c|}
\hline $\begin{array}{l}\text { Kerrick et al., } \\
2016 \text { [70] }\end{array}$ & USA & 121 & $\begin{array}{l}\text { Pre-post } \\
\text { study }\end{array}$ & Launch It & $\begin{array}{c}\text { Professional } \\
\text { s }\end{array}$ & $\begin{array}{l}\text { Networking, } \\
\text { entrepreneurship } \\
\text { concepts, } \\
\text { definition of } \\
\text { target markets, } \\
\text { market research, } \\
\text { concept } \\
\text { prototyping, } \\
\text { financial markets } \\
\text { and intellectual } \\
\text { property }\end{array}$ & 87 & 34 & $50-70$ & $\begin{array}{r}10 \text { weeks - trainer and } \\
30 \text { hours experts in the } \\
\text { community } \\
\text { (lawyers, etc.) }\end{array}$ \\
\hline
\end{tabular}

\begin{tabular}{|c|c|c|c|c|c|c|c|c|c|}
\hline $\begin{array}{c}\text { Kim et al., } \\
2020 \text { [71] }\end{array}$ & Korea & 1934 & $\begin{array}{c}\text { Quasi- } \\
\text { experimenta } \\
\text { l design }\end{array}$ & $\begin{array}{c}\text { KAIST Social } \\
\text { Entrepreneurshi } \\
\text { p MBA Program }\end{array}$ & $\begin{array}{l}\text { Secondary } \\
\text { education }\end{array}$ & $\begin{array}{l}\text { Social } \\
\text { entrepreneurial } \\
\text { skills }\end{array}$ & $30-60$ & 2 years & Teachers \\
\hline
\end{tabular}

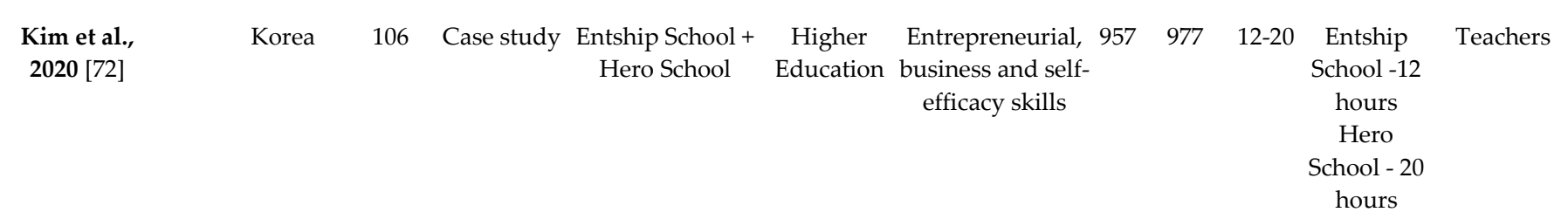

\begin{tabular}{|c|c|c|c|c|c|c|c|c|c|}
\hline $\begin{array}{c}\text { Klapper, } 2005 \\
\text { [73] }\end{array}$ & France & 83 & $\begin{array}{c}\text { Qualitative } \\
\text { design }\end{array}$ & $\begin{array}{c}\text { Project } \\
\text { Entreprendre }\end{array}$ & $\begin{array}{l}\text { Higher } \\
\text { Education }\end{array}$ & $\begin{array}{c}\text { Teamwork, } \\
\text { business plan, } \\
\text { interactivity, self- } \\
\text { confidence, } \\
\text { credibility, } \\
\text { balance between } \\
\text { formal and } \\
\text { informal. }\end{array}$ & $19-21$ & 5 months & $\begin{array}{l}\text { Teachers, } \\
\text { consultors }\end{array}$ \\
\hline
\end{tabular}

\begin{tabular}{|c|c|c|c|c|c|c|c|c|c|}
\hline $\begin{array}{l}\text { Kubberød et } \\
\text { al., } 2017 \text { [74] }\end{array}$ & Norway & 24 & $\begin{array}{c}\text { Qualitative } \\
\text { design }\end{array}$ & $\begin{array}{l}\text { The Norwegian } \\
\text { School of } \\
\text { Entrepreneurshi } \\
\text { p }\end{array}$ & $\begin{array}{l}\text { Higher } \\
\text { Education }\end{array}$ & $\begin{array}{l}\text { Entrepreneurial, } \\
\text { business and self- } \\
\text { efficacy skills }\end{array}$ & +23 & $\begin{array}{l}3 \text { months - } \\
48 \text { hours }\end{array}$ & Teachers \\
\hline
\end{tabular}

\begin{tabular}{|c|c|c|c|c|c|c|c|c|}
\hline $\begin{array}{l}\text { Lekoko et al., } \\
2012 \text { [75] }\end{array}$ & Botswana & 325 & Case study & $\begin{array}{c}\text { Entrepreneurshi } \\
\text { p Education }\end{array}$ & $\begin{array}{c}\text { Higher } \\
\text { Education }\end{array}$ & $\begin{array}{l}\text { Awareness that } \\
\text { entrepreneurship } \\
\text { education in } \\
\text { Botswana does } \\
\text { not develop } \\
\text { entrepreneurial } \\
\text { skills, which }\end{array}$ & $18-25$ & Teachers \\
\hline
\end{tabular}


makes it

impossible to

pursue a career

in the field of

entrepreneurship

\begin{tabular}{ccccccccc}
\hline $\begin{array}{c}\text { Lyons et al., } \\
2018 \text { [76] }\end{array}$ & USA & 335 & $\begin{array}{c}\text { Qualitative } \\
\text { design }\end{array}$ & Next 36 & $\begin{array}{c}\text { Secondary } \\
\text { education }\end{array}$ & $\begin{array}{c}\text { Increased } \\
\text { likelihood of } \\
\text { working or } \\
\text { founding a } \\
\text { startup }\end{array}$ & 0 & $\begin{array}{c}\text { Teachers, } \\
\text { entrepreneurs } \\
\text { funders }\end{array}$ \\
\hline
\end{tabular}

\begin{tabular}{|c|c|c|c|c|c|c|c|c|c|}
\hline $\begin{array}{l}\text { Mohamed et } \\
\text { al., } 2012 \text { [77] }\end{array}$ & Malaysia & 410 & $\begin{array}{c}\text { Qualitative } \\
\text { design }\end{array}$ & $\begin{array}{l}\text { Basic Student } \\
\text { Entrepreneurshi } \\
\text { p Program }\end{array}$ & $\begin{array}{l}\text { Higher } \\
\text { Education }\end{array}$ & $\begin{array}{l}\text { Skills to take } \\
\text { advantage of } \\
\text { business } \\
\text { opportunities, } \\
\text { marketing, } \\
\text { entrepreneurial } \\
\text { simulations and } \\
\text { analysis of the } \\
\text { characteristics of } \\
\text { successful } \\
\text { entrepreneurs }\end{array}$ & $18-40$ & $\begin{array}{l}6 \text { months - } \\
36 \text { hours }\end{array}$ & Teachers \\
\hline
\end{tabular}

\begin{tabular}{|c|c|c|c|c|c|c|c|c|c|c|c|}
\hline $\begin{array}{l}\text { Pedrini et al., } \\
\quad 2017 \text { [78] }\end{array}$ & Ghana & 30 & $\begin{array}{c}\text { Mixed } \\
\text { method } \\
\text { study design }\end{array}$ & E4impact MBA & $\begin{array}{l}\text { Higher } \\
\text { Education }\end{array}$ & $\begin{array}{c}\text { Business plan, } \\
\text { international } \\
\text { network of } \\
\text { partners and } \\
\text { investors }\end{array}$ & 25 & 5 & $27-49$ & $\begin{array}{l}12 \text { months } \\
-24 \text { hours }\end{array}$ & Teachers \\
\hline
\end{tabular}

\begin{tabular}{|c|c|c|c|c|c|c|c|c|c|c|c|}
\hline $\begin{array}{c}\text { Pepin, } 2018 \\
\text { [79] }\end{array}$ & Canada & 19 & Case study & $\begin{array}{c}\text { School Shop } \\
\text { Project }\end{array}$ & $\begin{array}{l}\text { Primary } \\
\text { education }\end{array}$ & $\begin{array}{c}\text { Experience of } \\
\text { what it means to } \\
\text { be an } \\
\text { entrepreneur }\end{array}$ & 9 & 10 & $7-8$ & $\begin{array}{c}\text { entire } \\
\text { school } \\
\text { year (from } \\
\text { September } \\
\text { to June) }\end{array}$ & Teachers \\
\hline
\end{tabular}

\begin{tabular}{|c|c|c|c|c|c|c|c|c|c|c|}
\hline $\begin{array}{l}\text { Peterman et } \\
\text { al., } 2003 \text { [80] }\end{array}$ & Australia & 236 & $\begin{array}{l}\text { Pre-post } \\
\text { study }\end{array}$ & $\begin{array}{c}\text { Young } \\
\text { Achievement } \\
\text { Australia }\end{array}$ & $\begin{array}{l}\text { Secondary } \\
\text { education }\end{array}$ & $\begin{array}{l}\text { Perception of the } \\
\text { benefits of } \\
\text { starting a } \\
\text { business; of the } \\
\text { benefits of EE } \\
\text { programs for } \\
\text { training potential } \\
\text { entrepreneurs as } \\
\text { a professional } \\
\text { career option }\end{array}$ & 90 & 146 & $15-18$ & $\begin{array}{l}9 \text { months - Teachers and } \\
36 \text { hours volunteers }\end{array}$ \\
\hline
\end{tabular}




\begin{tabular}{|c|c|c|c|c|c|c|c|c|c|c|c|}
\hline $\begin{array}{l}\text { Pinho et al., } \\
2019 \text { [81] }\end{array}$ & Portugal & 24 & Case study & UKids & $\begin{array}{l}\text { Primary } \\
\text { education }\end{array}$ & $\begin{array}{l}\text { Valuation of } \\
\text { individual } \\
\text { capacities, such } \\
\text { as creativity, self- } \\
\text { confidence, the } \\
\text { power of } \\
\text { argument, as } \\
\text { well as the } \\
\text { construction of } \\
\text { social skills, in } \\
\text { interpersonal and } \\
\text { group } \\
\text { relationships; } \\
\text { motivation to } \\
\text { work on public } \\
\text { causes in the } \\
\text { logic of } \\
\text { sustainable } \\
\text { development, } \\
\text { and openness to } \\
\text { new concepts, } \\
\text { such as } \\
\text { creativity, respect } \\
\text { for the } \\
\text { environment, } \\
\text { cooperation, } \\
\text { communication } \\
\text { of ideas. }\end{array}$ & 24 & 24 & $8-10$ & $\begin{array}{c}\text { entire } \\
\text { school } \\
\text { year }\end{array}$ & Teachers \\
\hline $\begin{array}{l}\text { Rigg et al., } \\
2020 \text { [82] }\end{array}$ & $\begin{array}{c}\text { Netherland } \\
\text { s }\end{array}$ & 8 & Pilot study & UKids & $\begin{array}{c}\text { Professional } \\
\text { s }\end{array}$ & $\begin{array}{l}\text { Social } \\
\text { entrepreneurial } \\
\text { skills }\end{array}$ & & & $18-25$ & 7 months & Teachers \\
\hline $\begin{array}{c}\text { San Tan et al., } \\
2006 \text { [83] }\end{array}$ & Singapore & & Pilot study & $\begin{array}{l}\text { Problem-Based } \\
\text { Learning }\end{array}$ & $\begin{array}{l}\text { Higher } \\
\text { Education }\end{array}$ & $\begin{array}{l}\text { Entrepreneurial } \\
\text { skills }\end{array}$ & & & $18-25$ & $\begin{array}{l}16 \text { weeks } \\
\text { in a } \\
\text { semester - } \\
32 \text { hours }\end{array}$ & Facilitator \\
\hline $\begin{array}{c}\text { Sánchez- } \\
\text { García \& } \\
\text { Hernández- } \\
\text { Sánchez, } 2016 \\
\text { [84] }\end{array}$ & Spain & 310 & $\begin{array}{l}\text { Quasi- } \\
\text { experimenta } \\
\text { l design }\end{array}$ & PREU & $\begin{array}{l}\text { Higher } \\
\text { Education }\end{array}$ & $\begin{array}{l}\text { Self-efficacy, } \\
\text { proactivity and } \\
\text { risk, finance, } \\
\text { marketing, } \\
\text { management; } \\
\text { skills such as } \\
\text { self-efficacy, } \\
\text { proactivity and } \\
\text { risk; interactive } \\
\text { practice with } \\
\text { entrepreneurs }\end{array}$ & 177 & 133 & $19-22$ & $\begin{array}{l}8 \text { months - } \\
28 \text { hours }\end{array}$ & Teachers \\
\hline
\end{tabular}




\begin{tabular}{|c|c|c|c|c|c|c|c|c|c|c|c|}
\hline $\begin{array}{c}\text { Santini et al., } \\
2020 \text { [85] }\end{array}$ & $\begin{array}{l}\text { Italy, } \\
\text { Germany } \\
\text { and } \\
\text { Slovenia }\end{array}$ & 41 & $\begin{array}{l}\text { Pre-post } \\
\text { study }\end{array}$ & Be the Change & $\begin{array}{c}\text { Professional } \\
\text { s }\end{array}$ & $\begin{array}{l}\text { Mentoring skills, } \\
\text { for example, } \\
\text { active listening } \\
\text { and guidance, } \\
\text { improving well- } \\
\text { being and self- } \\
\text { esteem, an } \\
\text { attitude of social } \\
\text { inclusion and } \\
\text { active aging. } \\
\text { Business and } \\
\text { socio-relational } \\
\text { skills, for } \\
\text { example, } \\
\text { benefiting from } \\
\text { the full } \\
\text { exploration of the } \\
\text { mentors' know- } \\
\text { how and their } \\
\text { relationship and } \\
\text { trust. }\end{array}$ & 41 & 33 & $\begin{array}{c}18-29 \mid \\
55-70\end{array}$ & $\begin{array}{c}\text { OAEs - } 16 \\
\text { hours of } \\
\text { training } \\
\text { Mentees - } \\
20 \\
\text { sessions } \\
\text { (40 hours) }\end{array}$ & $\begin{array}{c}\text { Mentors, } \\
\text { technical } \\
\text { experts in } \\
\text { education }\end{array}$ \\
\hline $\begin{array}{l}\text { Smith et al., } \\
2006 \text { [86] }\end{array}$ & $\begin{array}{c}\text { United } \\
\text { Kingdom }\end{array}$ & 16 & $\begin{array}{c}\text { Qualitative } \\
\text { design }\end{array}$ & $\begin{array}{c}\text { Discovering } \\
\text { Entrepreneurshi } \\
\text { p }\end{array}$ & $\begin{array}{l}\text { Higher } \\
\text { Education }\end{array}$ & $\begin{array}{c}\text { Extroversion, } \\
\text { taking risks, } \\
\text { tolerance of } \\
\text { ambiguity and } \\
\text { novelty, } \\
\text { independence, } \\
\text { leadership, } \\
\text { finding } \\
\text { opportunities, } \\
\text { creativity and } \\
\text { problem solving, } \\
\text { contacts and } \\
\text { social networks, } \\
\text { interpersonal } \\
\text { skills. }\end{array}$ & 8 & 8 & $18-25$ & $\begin{array}{c}10 \\
\text { sessions - } \\
20 \text { hours }\end{array}$ & Teachers \\
\hline $\begin{array}{l}\text { Soundarajan } \\
\text { et al., } 2016 \text { [87] }\end{array}$ & USA & 98 & $\begin{array}{l}\text { Quasi- } \\
\text { experimenta } \\
1 \text { design }\end{array}$ & Newpath & $\begin{array}{l}\text { Higher } \\
\text { Education }\end{array}$ & $\begin{array}{l}\text { Entrepreneurial } \\
\text { skills }\end{array}$ & & & $18-25$ & $\begin{array}{c}3 \text { weeks - } \\
\text { (campus } \\
\text { and visit } \\
\text { to Silicon } \\
\text { Valley) + } \\
12 \text { weeks } \\
\text { (internshi } \\
\text { p in a } \\
\text { company) } \\
-375 \\
\text { hours = } 15 \\
\text { weeks } 25 \\
\text { hours }\end{array}$ & $\begin{array}{c}\text { Teachers + } \\
\text { internship } \\
\text { supervisors + } \\
\text { local } \\
\text { businessmen }\end{array}$ \\
\hline
\end{tabular}




\begin{tabular}{|c|c|c|c|c|c|c|c|c|}
\hline $\begin{array}{l}\text { Ulvenblad et } \\
\text { al., } 2020 \text { [88] }\end{array}$ & Sweden & 109 & $\begin{array}{c}\text { Mixed } \\
\text { method } \\
\text { study design }\end{array}$ & $\begin{array}{l}\text { Leader Practice + } \\
\text { Lean Agriculture }\end{array}$ & $\begin{array}{c}\text { Professional } \\
\mathrm{s}\end{array}$ & $\begin{array}{l}\text { Self-leadership } \\
\text { and team } \\
\text { leadership, } \\
\text { delegation of } \\
\text { tasks, } \\
\text { communication } \\
\text { with employees } \\
\text { and family, work } \\
\text { routines, time } \\
\text { management. }\end{array}$ & $\begin{array}{c}50+53 \\
\text { years - } \\
\text { averag } \\
\text { e }\end{array}$ & $\begin{array}{c}\text { Trainers and } \\
\text { Coaches }\end{array}$ \\
\hline
\end{tabular}

\begin{tabular}{|c|c|c|c|c|c|c|c|c|c|c|c|}
\hline $\begin{array}{c}\text { Wu et al., } 2018 \\
\text { [89] }\end{array}$ & Taiwan & 21 & $\begin{array}{c}\text { Mixed } \\
\text { method } \\
\text { study design }\end{array}$ & PowToon & $\begin{array}{l}\text { Higher } \\
\text { Education }\end{array}$ & $\begin{array}{l}\text { Perception that } \\
\text { animated } \\
\text { presentations } \\
\text { attracted more } \\
\text { investment; } \\
\text { creating videos } \\
\text { helped the team } \\
\text { better present } \\
\text { their business } \\
\text { ideas to } \\
\text { investors; } \\
\text { whoever } \\
\text { generates a } \\
\text { business idea } \\
\text { does not } \\
\text { necessarily } \\
\text { influence } \\
\text { investor } \\
\text { decisions. }\end{array}$ & 25 & 20 & +23 & $\begin{array}{c}\text { EMBA - } 36 \\
\text { hours }\end{array}$ & Teachers \\
\hline $\begin{array}{c}\text { Wu et al., } 2019 \\
\text { [90] }\end{array}$ & Taiwan & 32 & $\begin{array}{l}\text { Qualitative } \\
\text { design }\end{array}$ & MOOCs course & $\begin{array}{l}\text { Higher } \\
\text { Education }\end{array}$ & $\begin{array}{c}\text { Social } \\
\text { entrepreneurship } \\
\text { courses with a } \\
\text { mixed approach } \\
\text { can be used } \\
\text { effectively to } \\
\text { help students } \\
\text { achieve different } \\
\text { levels of teaching } \\
\text { objectives in the } \\
\text { affective domain, } \\
\text { which is a } \\
\text { lengthy process, } \\
\text { especially at } \\
\text { higher education } \\
\text { levels. }\end{array}$ & 12 & 20 & 21-24 & $\begin{array}{c}\text { 9-week } \\
\text { course - } 18 \\
\text { hours }\end{array}$ & Teachers \\
\hline
\end{tabular}

The participants in the studies were aged between 7 and 70 years old, with the percentage about $48 \%$ being between 7 and 17 years old, and about $52 \%$ being between 18 and 70 years old. In addition, it was found that $2 \%$ of the total participants attended primary 
school, 20\% secondary education, 33\% secondary education, 35\% higher education and $10 \%$ were professionals.

Some studies did not show the differentiation between genders, and only $68 \%$ indicated it explicitly. Among the studies that reported genders, $45 \%$ of the participants in these studies were male and 55\% were female.

The recipients of the programs of the 29 studies were distributed across all levels of education, highlighting higher education (59\%), professionals with $21 \%$ of participants, $7 \%$ of secondary education, $7 \%$ of primary education, $3 \%$ of middle and primary education, and $3 \%$ of middle and secondary education.

Regarding design, the studies varied between qualitative studies (31\%), case studies $(21 \%)$, mixed studies $(17 \%)$, quasi-experimental designs $(14 \%)$, pre and post test design $(10 \%)$ and pilot study $(7 \%)$.

In terms of duration, the programs reported in the different studies vary between 18 hours [90], 24 hours [67,78], 36 hours [69] and 924 hours [66], corresponding to courses taught between two months, a semester, a year or two years.

These studies are focused on programs that specifically target the development of ESs $[62,63,65,66,87]$, the development of business plans and models [73,74,78,91], the promotion of skills within the scope of social entrepreneurship and sustainability $[64,81,82]$, in the scope of agriculture [77,88], of technologies $[63,87,90]$. Some specific competences also stand out, such as teamwork [63,67,73,81,86,89], self-efficacy $[64,70,72,74,78,84]$, leadership $[86,88]$, self-confidence $[64,73,81]$, proactivity and initiative $[64,66,84]$, innovation $[64,66,68,84]$, problem solving $[66,72,86]$, empathy $[64,81]$, selfesteem [85] and time management [88].

Most studies had teachers as the only facilitators of the programs (66\%) (n=19), but in addition to teachers, the studies presented the collaboration of local entrepreneurs [76,87], volunteers [80], mentors and coaches [85.88], professional internships supervisors [87], community specialists such as lawyers and military personnel [70].

The pedagogical activities presented in the studies were quite diverse, with emphasis on classes, lectures, workshops, business simulations, group dynamics and games, visits to companies, internship experiences in incubators, simulating the early stages of a startup. However, it was found that in the early stage of education, there were activities such as the creation of a market in the school [79], storytelling and mindfulness [64] and educational games [82]. In turn, in secondary and higher education, activities were carried out to create, develop and implement an entrepreneurial project, using business models $[73,78,80]$, marketing studies $[71,77,80,84]$ and finance $[70,80]$.

For the evaluation of the programs, the studies presented several instruments, highlighting interviews as focus groups and the questionnaires elaborated for the evaluation of the effectiveness of the programs. But specific questionnaires were used for each program, such as: ATE test [92], which measures students' entrepreneurial potential or attitude; EP Scale [93], which measures entrepreneurial passion; COE questionnaire (Entrepreneurial Orientation Questionnaire) [94].

The theoretical models on which the study interventions were based are very diverse, naturally combining these theoretical approaches with the pedagogical resources and techniques of entrepreneurial pedagogy. Among the theoretical models we highlight those of the theory of planned behaviour [95], the theory of social learning [96], of selfefficacy [97], of passion as a key to entrepreneurial action [93], of the entrepreneurial ecosystem [71] and collaborative learning [98].

\subsection{Methodological quality assessment}

Considering the four major types of study (case reports, case control, quasi-experimental studies, and qualitative studies), we followed the JBI criteria. And we evaluate the items of each of these four checklists, with the respective scale (No, Yes, Unclear, Not applicable). As such, it is concluded that all studies met the inclusion quality criteria. 
Regarding the case study and pilot study $(\mathrm{n}=8 \mid 28 \%)$, on average, $73 \%$ of the criteria were met. It should be noted that the less clear items were those of demographic characteristics as well as the adverse effects of the intervention, which were not properly described. In qualitative studies $(\mathrm{n}=9 \mid 31 \%)$, on average, $80 \%$ of the criteria were fully met. The item with the lowest score was the sample's representativeness. Also, some of the studies do not report how the interviews were conducted. In quasi-experimental studies ( $\mathrm{n}=4 \mid 13 \%)$, the criteria were fully met at $100 \%$, which explains the value of this type of study for interventions through skills development programs. In turn, in the mixed-method study design and pre-post study ( $\mathrm{n}=8 \mid 28 \%$ ), the criteria were fully met at $66 \%$, and among the remaining $26 \%$ the criteria were not met and $8 \%$ did not apply. In addition, there was a lack of explanation of ethical issues in several articles.

\subsection{Results of interventions}

The results achieved by the programs are diverse, revealing the multiplicity of objectives, teaching methods, target groups and contexts.

The quasi-experimental studies, mixed-method study design, pre-post study $[63,65,67,70,71,78,80,84,85,87-89]$ revealed consistent statistical results on the gains achieved with the interventions, and there were global improvements between pre and post-intervention. However, the qualitative designs [62,64,68,74,76,77,86,90,99], the case studies and pilot studies also showed, through the data obtained in the interviews and focus groups, that there were improvements in the participants in terms of the acquisition of ESs, which demonstrates that the evaluation of entrepreneurial skills training must be carried out not only through statistical analysis, but also with content analysis and the triangulation of these types of analyses.

Gains can be grouped into three major thematic groups: entrepreneurial skills (69\%), for example - NewPath [87], Standup [62], EME, EJE and ÍCARO [63]; business management $(38 \%)$ - for example Launch It [70], PREU [84], Project Start [66]; and social entrepreneurship (10\%) - for example UKids [82], Kaist [71] and MOOC [90]. It should be noted that some of these studies deal with more than one thematic area, as shown in the following examples.

The Next 36 program (N36) demonstrates how participating in a program significantly increases the subsequent likelihood that a finalist will work at a startup, either as a founder or as a collaborator [76].

The School Shop Project program (SSP) shows how students learn what it means to be an entrepreneur, both through the processes of reflection from the questions of the inquiry and the processes of investigation, which allows to know this domain and the development of the skills of dialogue and critical reflection [79].

The Discovering Entrepreneurship program (DE) brought results regarding motivation, ESs and the creation of new companies. In this sense, it motivated students to be more expansive, to have greater clarity on the value of contributing to the community, to take risks, to focus themselves on the satisfaction of their achievements, for rebellion, to have self-control, high tolerance to ambiguity and to novelty, independence and autonomy, but with a focus on people and the search for challenges. In turn, the developed ESs were: social skills, leadership in networking, coaching and mentoring, identifying opportunities, creativity and creative problem solving. Finally, in terms of the creation of new companies, there were gains in establishing contacts through networking, risk-taking and interpersonal skills [86].

The Leader Practice program (LP) revealed gains in self-leadership and the role of leader; in addition, students achieved gains in understanding the fact that leaders can delegate tasks and responsibilities, and recognised the need for continuous improvement in communication with employees and with their family members; in turn, the Lean 
Agriculture program (LA) proved to be effective in discovering ways of working smoothly, establishing work routines and following previously established procedures, and managing time in a correct way [88].

The Project Entreprendre program (PE) proved to be useful in achieving results in the following areas: teamwork, business plan development, interactivity in project development, demand for continuous improvement, self-confidence, empowerment, attention to credibility, and seeking a balance between the formal and the informal [73]. Most studies have shown positive results from the interventions. However, it is worth highlighting a case in which the programs used in primary and middle education did not fully reach the objectives initially defined [63], this fact being justified by the authors with the possibility that they did not properly elaborate what was intended to be specifically achieved with this entrepreneurship education action and/or the fact that the design of the evaluation strategies was not the most appropriate.

\section{Discussion}

Regarding the effectiveness of the EEP, this systematic review provided an overview of the current literature, analysing 29 programs, from 24 countries. The results suggested that approaches focused on ESs had a significant effect on the promotion of a certain type of culture, as is clearly shown in the UKids initial teacher training program, which aims to establish entrepreneurship, especially social entrepreneurship, as an element of teaching in primary schools [82]. This option has also been followed in other contexts, such as in Brazil, Portugal and Sweden [100-102].

As suggested by Lyons and Zhang [2018], the programs prove to be more effective for those who have limited access to entrepreneurial opportunities. Thus, they are more beneficial to participants who, otherwise, would have more difficulty in accessing the resources and skills of the programs. For this reason, the dissemination of this culture among all students becomes very significant, in the logic of an inclusive education that promotes social ascension [103-107].

In this review, there was a tendency for the EEPs to have a digital format, as is clearly shown in Newpath [87], for the development of projects in the scope of social entrepreneurship [38,71,82].

Among the strategies that stand out are those that lead to the promotion of communication skills for an adequate presentation of products and services $[62,73,89,108]$, in agreement with McCollough et al. [109] which advocates a streamlining of the model for the rapid presentation of ideas and the effective presentation strategies discussed by Jardim and Silva (2019), namely the importance to be given to the management of social networks, the use of digital platforms and communication channels best suited to a particular customer segment. It has also been shown that networking is crucial in EE, with particular attention to partnerships established with institutions outside the school itself, which is in line with Cheung's research in the context of secondary education in Hong Kong [9]. In summary, and based on the review carried out, a flowchart was drawn up with the main conclusions (cf. Figure 2), highlighting the problems emerging in the current socio-educational context, such as the high rate of unemployment, ecological and environmental issues, and academic and academic failure. Considering this contextual complexity, most of the reviewed programs were developed based on the definition of a unique identity, pedagogy and evaluation, but always aiming to educate for the development of a culture where the capacity to create value is predominant. Among the identified entrepreneurial skills, we find spirit of initiative, innovation and problem solving, global leadership, teamwork and networking, digital and communication skills, the use and creation of business models, the use of marketing and e-commerce. We concluded that the effectiveness of the EEP is mainly due to the quality of the design of the programs themselves, proven, for example, by their pedagogical approach [teaching 
methods, excellence of the facilitator and activities carried out]; by the predispositions of the participants, that is, if they started working on entrepreneurial skills since childhood, it becomes much more natural to be an entrepreneur; and by their integration or not in entrepreneurial ecosystems, verifying that whoever was born and lived in an entrepreneurial family or regional ecosystem, more easily takes chances to innovate. Thus, the promotion of an entrepreneurial culture is the consequence of the combination of these elements, mobilizing individuals and societies for a continuous creation in this global world. For all these reasons, it is necessary to take advantage of the opportunities and face the current challenges, namely those related to the integration of EE in all levels of education, but giving priority to those aimed at younger generations, as well as seeking to improve the quality of scientific production in this field. For this, it is necessary to carry out, with rigor and methodological quality, empirical studies, as well as to develop differentiated and specific pedagogies suitable for students from pre-school to higher education.

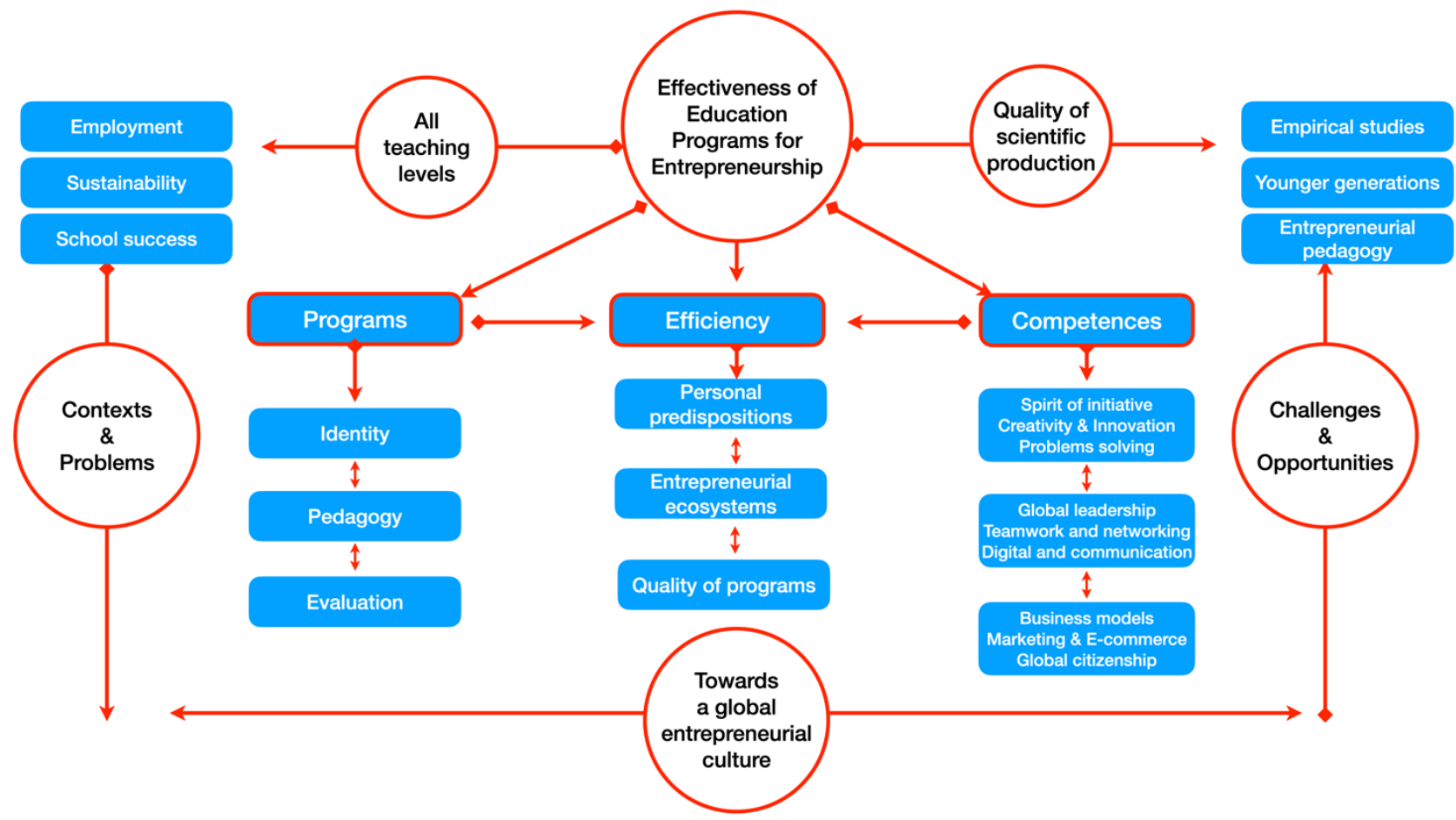

Figure 2. Flowchart with the simplified integration of the main results of the review and their implications for future studies.

Despite the results obtained, this review is not exempt from limitations. Thus, the characteristics of the studies included here vary widely. For example, regarding the main focus of the intervention, study design, number of sessions, delivery resources and sample size. For this reason, meta-analyses and statistical comparisons were not possible. In addition, by restricting the literature search to include only articles published in scientific journals in the past two decades, relevant publications such as conference papers, and dissertations may have been lost. Finally, although the methodological rigor of the studies has been assessed, it is important to note that this only indicates the overall quality of each study, including details of the program, but not specifically how information and coping strategies were addressed in the intervention.

In the continuation of this study, we propose that the EEP must be designed considering both the intervention and the research, which will allow to the collection and analyse of results with the methodological rigor necessary for an improvement in education. In 
addition, we suggest carrying out comparative studies of entrepreneurial pedagogy, such as the effectiveness of entrepreneurship teaching methods used by different facilitators and teachers.

\section{Conclusion}

This study demonstrated that there has been a steady increase in EEP, and the vast majority of these programs are aimed at students in higher education, a few in secondary education, and even less in basic and pre-school education. In addition, it has been shown that most studies are more conceptual than empirical, thus neglecting cognitive and behavioural results. When their effectiveness was assessed empirically, the EEP revealed a moderate effect in terms of activating entrepreneurial intent and a more notable effect on the development of ESs. It was also found that, more than the EEP, it is the personal predispositions and family, economic and cultural contexts that most influence the option for the development of entrepreneurial projects.

ESs were targeted at all levels of education and in all studies, however, greater attention was paid to these competencies in basic and secondary education. And the results of the studies show the effectiveness of these programs in promoting self-efficacy, an entrepreneurial attitude, resilience, risk-taking and openness to novelty. In turn, there is no increase in the intention to create the business itself, since this intention is determined by predisposition, namely socio-cultural and family aspects.

The challenges that entrepreneurial leaders have in the current context are also noted, clearly marked by technologies and remote work, by the social complexity and unpredictability of work, by the need to reconcile health and economy, entrepreneurship and citizenship. And one of the privileged strategies in this area is the educator himself. His active, inspiring and differentiating presence in the most diverse promotional contexts of entrepreneurship will allow the development of entrepreneurial culture values. They should be skilled in the use of these and other useful tools for the promotion of an entrepreneurial mindset.

Thus, this systematic review on the EEP points to the need for this type of programs to be applied preferentially since the early school years, since it is at that time that the predispositions for the development of entrepreneurial skills and intentions are created. This condition is corroborated by the global geography of the EEP which demonstrates that, where there is currently an entrepreneurial culture, countries have made a long educational journey, with strategic options from the point of view of educational policies that defend entrepreneurship among the younger generations.

\section{References:}


1. Jardim J. Empreende: Manual Global de Educação para o Empreendedorismo [Empreende: Global Entrepreneurship Education Handbook]. Lisboa, Portugal: Theya Editores; 2021.

2. Jardim J, Franco JE. Empreendipédia - Dicionário de Educação para o Empreendedorismo. Lisboa: Gradiva; 2019.

4. Jardim J. Regiões Empreendedoras: Descrição e avaliação dos contextos, determinantes e políticas favoráveis à sua evolução. Rev Divulg Científica AICA. 2020;12(1):197-212.

5. Porfírio JA, Carrilho T, Felício JA, Jardim J. Leadership characteristics and digital transformation. J Bus Res. 2021 Nov;124:610-9.

6. Byun C-G, Sung C, Park J, Choi D. A Study on the Effectiveness of Entrepreneurship Education Programs in Higher Education Institutions: A Case Study of Korean Graduate Programs. J Open Innov Technol Mark Complex. 2018 Jun $28 ; 4(3): 26$.

7. Carree M, Della Malva A, Santarelli E, Carree M, Malva AD, Santarelli E. The contribution of universities to growth: empirical evidence for Italy. J Technol Transf. 2011;39:393-414.

8. Hernández-Sánchez BR, Sánchez-García JC, Mayens AW. Impact of Entrepreneurial Education Programs on Total Entrepreneurial Activity: The Case of Spain. Adm Sci. 2019 Mar 13;9(25):1-16.

9. Cheung CK. Entrepreneurship education in Hong Kong's secondary curriculum: Possibilities and limitations. Educ Train. 2008;50(6):500-15.

10. Abdelkarim A. Toward Establishing Entrepreneurship Education and Training Programmes in a Multinational Arab University. J Educ Train Stud. 2018 Dec 3;7(1):1.

11. Basu R. Entrepreneurship Education in India: A Critical Assessment and a Proposed Framework. Technol Innov Manag Rev. 2014 Aug 27;4(8):5-10.

12. Vang J. Entrepreneurship in Western Europe: a contextual perspective. Eur Plan Stud. 2017;25(6):1099-100.

13. Solomon G. An examination of entrepreneurship education in the United States. J Small Bus Enterp Dev. 2007 May $22 ; 14(2): 168-82$.

14. Valerio A, Parton B, Robb A. Entrepreneurship Education and Training Programs around the World: Dimensions for Success. World Bank Publications. Washington: The World Bank; 2014.

15. Nieuwenhuizen C, Groenewald D, Davids J, Van Rensburg LJ, Schachtebeck C. Best practice in entrepreneurship education. Probl Perspect Manag. 2016;14(3):528-36.

16. Cooper A. Entrepreneurship: The Past, the Present, the Future. In: Handbook of Entrepreneurship Research. SpringerVerlag; 2005. p. 21-34.

17. Landström H. The Evolution of Entrepreneurship as a Scholarly Field. Found Trends® Entrep. 2020;16(2):65-243.

18. Katz JA. The chronology and intellectual trajectory of American entrepreneurship education. J Bus Ventur. 2003 Mar;18(2):283-300.

19. European Commission. Entrepreneurship Education in Europe: Fostering Entrepreneurial Mindsets through Education and Learning. European Union; 2006.

20. Krueger N. Entrepreneurial Education in Practice: Part 1 - The Entrepreneurial Mindset. Paris: Secretary-General of the OECD; 2015. 
21. Comissão Europeia. Fomentar a Promoção das Atitudes e Competências Empresariais no Ensino Básico e Secundário. Bruxelas; 2004.

22. Römer-Paakkanen T, Suonpää M. Multiple Objectives and Means of entrepreneurship education at Finnish universities of applied sciences. 2017.

23. Plourde H, Pelletier D. Introduction to Entrepreneurial Culture. Québec: Gouvernement du Québec - Ministère de l'Éducation, du Loisir et du Sport; 2007. 80 p.

24. Mwasalwiba ES, Samwel Mwasalwiba E, Mwasalwiba ES. Entrepreneurship education: A review of its objectives, teaching methods, and impact indicators. Educ Train. 2010;52(1):20-47.

25. Jardim J. Programa de Desenvolvimento de Competências Pessoais e Sociais: Estudo para a Promoção do Sucesso Académico. (Lisboa, Portugal): Instituto Piaget; 2010.

26. Dolabela F. O segredo de Luísa - Uma ideia, uma paixão e um plano de negócios: como nasce o empreendedor e se cria uma empresa. São Paulo: Editora de Cultura; 2006.

27. Dolabela F. Oficina do Empreendedor. São Paulo: Editora de Cultura; 1999.

28. Cárcamo-Solís M de L, Arroyo-López M del P, Alvarez-Castañón L del C, García-López E. Developing entrepreneurship in primary schools. The Mexican experience of "My first enterprise: Entrepreneurship by playing." Teach Teach Educ. 2017 May 1;64:291-304.

29. Barba-Sánchez V, Atienza-Sahuquillo C. The development of entrepreneurship at school: the Spanish experience. Educ Train. 2016 Aug 8;58(7-8):783-96.

30. Jardim J, Soares JH, Moutinho A, Calheiros C, Cardoso P, Cardoso MS, et al. Brincadores de Sonhos. Lisboa: Theya; 2015.

31. Jardim J, Rodrigues R, Gouveia T, Pereira M, Gomes F, Paolineli LA, et al. Exploradores de Sonhos. Lisboa: Theya; 2018.

32. Hercz M, Pozsonyi F, Flick-Takács N. Supporting a Sustainable Way of Life-Long Learning in the Frame of Challenge-Based Learning. Discourse Commun Sustain Educ. 2021 Jan 13;11(2):45-64.

33. Kirkley WW. Cultivating entrepreneurial behaviour: entrepreneurship education in secondary schools. Asia Pacific J Innov Entrep. 2017 Apr 3;11(1):17-37.

34. Steenekamp AG, Van der Merwe SP, Athayde R. An investigation into youth entrepreneurship in selected South African secondary schools: An exploratory study. South African Bus Rev. 2011;15(3):46-75.

35. Jardim J, Lima J, Grilo C. Os Originais: Programa de Empreendedorismo Social com Jovens [The Originals: Program of Social Entrepreneruship with Youth]. Lisboa: Theya; 2019.

36. Karlsson H. Summer Entrepreneur an Activity for stimulating Entrepreneurship Among Youths: A Case Study in a Swedish County. US-China Educ Rev. 2011;1(5):715-25.

37. Audretsch DB, Obschonka M, Gosling SD, Potter J. A new perspective on entrepreneurial regions: linking cultural identity with latent and manifest entrepreneurship. Small Bus Econ. 2017 Mar 1;48(3):681-97.

38. Forcher-Mayr M, Mahlknecht S. A Capability Approach to Entrepreneurship Education: The Sprouting Entrepreneurs Programme in Rural South African Schools. Discourse Commun Sustain Educ. 2020 Jun 1;11(1):119-33. 
39. Cumberland DM. Training and Educational Development for "Vetrepreneurs." Adv Dev Hum Resour. 2017 Feb 22;19(1):88-100.

40. Alalwany H, Saad F. Entrepreneurial education programmes and their impact on entrepreneurs' attributes. Proc Eur Conf Innov Entrep ECIE.2015-Janua:15-24.

41. Bell R. Unpacking the link between entrepreneurialism and employability: An assessment of the relationship between entrepreneurial attitudes and likelihood of graduate employment in a professional field. Educ Train. 2016 Jan $11 ; 58(1): 2-17$.

42. Morris MH, Kuratko DF. Building university 21st century entrepreneurship programs that empower and transform. Adv Study Entrep Innov Econ Growth. 2014;24:1-24.

43. Gibb A, Price A. A Compendium of Pedagogies for Teaching Entrepreneurship. International Entrepreneurship Educators Programme. IEEP and NCEE; 2014. $218 \mathrm{p}$.

44. Bacigalupo M, Kampylis P, Punie Y, Van den Brande G. EntreComp: the entrepreneurship competence framework. Luxembourg: Publication Office of the European Union; 2016. 39 p.

45. Czyzewska M, Mroczek T. Data Mining in Entrepreneurial Competencies Diagnosis. Educ Sci. 2020 Jul 28;10(8):196.

46. Hebles M, Llanos-Contreras O, Yániz-Álvarez-De-Eulate C. Perceived evolution of the entrepeneurial competence based on the implementation of a training program in entrepreneurship and innovation. Rev Esp Orientac y Psicopedag. 2019 Jul 1;30(1):9-26.

47. Jardim J. Competências empreendedoras. In: Jardim J, Franco JE, editors. Portugal Empreendedor: Trinta Figuras Empreendedoras da Cultura Portuguesa - Relevância dos Modelos para a Promoção do Empreendedorismo. Lisboa: Imprensa Nacional Casa da Moeda; 2013. p. 69-79.

48. Jardim J, Pereira A, Vagos P, Direito I, Galinha SA. The Soft Skills Inventory: Construction procedures and psychometric analysis. Psychol Rep. 2020; 0(0), 1-29.

49. Jardim J. 10 Competências Rumo à Felicidade: Guia Prático para Pessoas, Equipas e Organizações Empreendedoras. 2. ${ }^{\text {. Lisboa, }}$ Portugal: Instituto Piaget; 2012.

50. Vega-Gómez FI, Miranda González FJ, Chamorro Mera A, Pérez-Mayo J. Antecedents of Entrepreneurial Skills and Their Influence on the Entrepreneurial Intention of Academics. SAGE Open. 2020 Apr 3;10(2):215824402092741.

51. RezaeiZadeh M, Hogan M, O’Reilly J, Cunningham J, Murphy E. Core entrepreneurial competencies and their interdependencies: insights from a study of Irish and Iranian entrepreneurs, university students and academics. Int Entrep Manag J. 2017 Mar 1;13(1):35-73.

52. Quieng MC, Lim PP, Lucas MRD. 21st Century-based Soft Skills: Spotlight on Non-cognitive Skills in a Cognitiveladen Dentistry Program. Eur J Contemp Educ. 2015;11(1):72-81.

53. European Commission. Entrepreneruship Education: A Guide for Educators. EU Commission - Entrepreneurship 2020 Unit. Brussels: Directorate-General for Enterprise and Industry; 2014.

54. Politańska J. Best Practices in Teaching Entrepreneurship and Creating Entrepreneurial Ecosys. Warszawa: Fundacja Światowego Tygodnia Przedsiębiorczości; 2014.

55. Ashoka U, Brock DD. Social Entrepreneurship Education Resource Handbook. Haryana, Índia: Ashoka; 2011. 
56. Moher D, Shamseer L, Clarke M, Ghersi D, Liberati A, Petticrew M, et al. Preferred reporting items for systematic review and meta-analysis protocols (PRISMA-P) 2015 statement. Rev Esp Nutr Humana y Diet. 2016;20(2):148-60.

57. The Joanna Briggs Institute (JBI). Checklist for quasi-experimental (Non-randomized experimental studies). Australia: Joanna Briggs Institute; 2017.

58. The Joanna Briggs Institute (JBI). Checklist for qualitative research. Australia: Joanna Briggs Institute; 2020.

59. The Joanna Briggs Institute (JBI). Checklist for randomized controlled trials. Australia: Joanna Briggs Institute; 2020.

60. Bártolo A, Santos IM, Monteiro S. Toward an Understanding of the Factors Associated With Reproductive Concerns in Younger Female Cancer Patients. Cancer Nurs. 2020;Publish Ah(0):1-13.

61. Moher D, Liberati A, Tetzlaff J, Altman DG, Altman D, Antes G, et al. Preferred reporting items for systematic reviews and meta-analyses: The PRISMA statement. PLoS Med. 2009 Jul;6(7):1-6.

62. Backs S, Schleef M, Buermann HW. Stand up-it's all about the team? The composition of entrepreneurial teams in entrepreneurship education at a German university. J Entrep Educ. 2019;22(4):1-20.

63. Bernal Guerrero A, Cárdenas Gutiérrez AR. Evaluación del potencial emprendedor en escolares. Una investigación longitudinal. Educ XX1. 2017 May 29;20(2):73-94.

64. Bisanz A, Hueber S, Lindner J, Jambor E. Social Entrepreneurship Education in Primary School: Empowering Each Child with the YouthStart Entrepreneurial Challenges Programme. Discourse Commun Sustain Educ. 2020 Jan $8 ; 10(2): 142-56$.

65. Boldureanu G, Ionescu AM, Bercu A-MM, Bedrule-Grigorut MV, Boldureanu D, Bedrule-Grigoruţă MV, et al. Entrepreneurship Education through Successful Entrepreneurial Models in Higher Education Institutions. Sustainability. 2020 Feb 10;12(3):1-33.

66. Dominguinhos PMC, Carvalho LMC. Promoting business creation through real world experience: Projecto Começar. Educ Train. 2009;51(2):150-69.

67. Fayolle A, Gailly B. Évaluation d'une formation en entrepreneuriat: Prédispositions et impact sur l'intention d'entreprendre. Management. 2009;12(3):175-203.

68. Hebles M, Llanos-Contreras O, Yániz-Álvarez-de-Eulate C. Evolución percibida de la competencia para emprender a partir de la implementación de un programa de formación de competencias en emprendimiento e innovación. REOP - Rev Española Orientación y Psicopedag. 2019;30(1):9.

69. Heinonen J, Poikkijoki SA, Vento-Vierikko I. Entrepreneurship for Bioscience Researchers: A Case Study of an Entrepreneurship Programme. Ind High Educ. 2007;21(1):21-30.

70. Kerrick SA, Cumberland DM, Choi N. Comparing military veterans and civlians responses to an Entrepreneurship education program. J Entrep Educ. 2016;19(1):9-23.

71. Kim MG, Lee JH, Roh T, Son H. Social entrepreneurship education as an innovation hub for building an entrepreneurial ecosystem: The case of the KAIST social entrepreneurship MBA program. Sustain. 2020;12(22):1-23.

72. Kim G, Kim D, Lee WJ, Joung S. The Effect of Youth Entrepreneurship Education Programs: Two Large-Scale Experimental Studies. SAGE Open. 2020 Jul 1;10(3):215824402095697.

73. Klapper R. Training entrepreneurship at a French grande école: The Projet Entreprendre at the ESC Rouen. J Eur Ind Train. 2005 Dec 1;29(9):678-93. 
74. Kubberød E, Pettersen IB. Exploring situated ambiguity in students' entrepreneurial learning. Educ Train. 2017;59(3):265-79.

75. Lekoko M, Rankhumise EM, Ras P. The effectiveness of entrepreneurship education: What matters most? African J Bus Manag. 2012 Dec 26;6(51):12023-32.

76. Lyons E, Zhang L. Who does (not) benefit from entrepreneurship programs? Strateg Manag J. 2018 Jan 1;39(1):85112.

77. Mohamed Z, Rezai G, Shamsudin MN, Mahmud MM az. Enhancing young graduates' intention towards entrepreneurship development in Malaysia. Educ Train. 2012 Sep;54(7):605-18.

78. Pedrini M, Langella V, Molteni M. Do entrepreneurial education programs impact the antecedents of entrepreneurial intention?: An analysis of an entrepreneurship MBA in Ghana. J Enterprising Communities. 2017;11(3):373-92.

79. Pepin M. Learning to be enterprising in school through an inquiry-based pedagogy. Ind High Educ. 2018;32(6):41829.

80. Peterman NE, Kennedy J. Enterprise Education: Influencing Students' Perceptions of Entrepreneurship. Entrep Theory Pract. 2003 Mar;28(2):129-44.

81. Pinho MI, Fernandes D, Serrão C, Mascarenhas D. Youth Start Social Entrepreneurship Program for Kids: Portuguese UKIDS-Case Study. Discourse Commun Sustain Educ. 2019;10(2):33-48.

82. Rigg E, van der Wal-Maris S. Student Teachers' Learning About Social Entrepreneurship Education - A Dutch Pilot Study in Primary Teacher Education. Discourse Commun Sustain Educ. 2020 Jun 1;11(1):41-52.

83. San Tan S, Ng CKF. A problem-based learning approach to entrepreneurship education. Educ + Train. 2006 Jul $1 ; 48(6): 416-28$.

84. Sánchez-García JC, Hernández-Sánchez B. Influencia del Programa Emprendedor Universitario (PREU) para la mejora de la actitud emprendedora. PAMPA. 2016 Aug 25;(13):55-75.

85. Santini S, Baschiera B, Socci M. Older adult entrepreneurs as mentors of young people neither in employment nor education and training (NEETs). Evidences from multi-country intergenerational learning program. Educ Gerontol. 2020 Mar 3;46(3):97-116.

86. Smith AJ, Collins LA, Hannon PD. Embedding new entrepreneurship programmes in UK higher education institutions: Challenges and considerations. Educ + Train. 2006 Oct 1;48(8-9):555-67.

87. Soundarajan N, Camp SM, Lee D, Ramnath R, Weide BW. NEWPATH: An innovative program to nurture IT entrepreneurs. Adv Eng Educ. 2016;5(1):1-27.

88. Ulvenblad P, Barth H, Ulvenblad P-O, Ståhl J, Björklund JC. Overcoming barriers in agri-business development: two education programs for entrepreneurs in the Swedish agricultural sector. J Agric Educ Ext. 2020 Oct 19;26(5):443-64.

89. Wu YJ, Yuan C-H, Pan C-I. Entrepreneurship Education: An Experimental Study with Information and Communication Technology. Sustainability. 2018;10(3):1-13.

90. Wu WH, Kao HY, Wu SH, Wei CW. Development and evaluation of affective domain using student's feedback in entrepreneurial Massive Open Online Courses. Front Psychol. 2019 May 24;10(MAY):1109.

91. White RE, Thornhill S, Hampson E. Entrepreneurs and evolutionary biology: The relationship between testosterone and new venture creation. Organ Behav Hum Decis Process. 2006;100(1):21-34. 
92. Athayde R. Measuring enterprise potential in young people. Entrep Theory Pract. 2009;33(2):481-500.

93. Cardon MS, Gregoire DA, Stevens CE, Patel PC. Measuring entrepreneurial passion: Conceptual foundations and scale validation. J Bus Ventur. 2013;

94. Sánchez-García JC. Evaluación de la personalidad emprendedora: Validez factorial del cuestionario de orientación emprendedora (COE). Rev Latinoam Psicol. 2010;42(1):41-52.

95. Ajzen I. The theory of planned behavior. Organ Behav Hum Decis Process. 1991;50(2):179-211.

96. Bandura A. Social learning theory. New York, US: General Learning Press; 1971.

97. Bandura A. Self-efficacy: the exercise of control. Vol. 35, Choice Reviews Online. Freeman and Company; 1997.

98. Dillenbourg P, Traum D. Sharing solutions: Persistence and grounding in multimodal collaborative problem solving. J Learn Sci. 2006;15(1):121-51.

99. Klapper R. Training entrepreneurship at a French grande école. J Eur Ind Train. 2005 Dec;29(9):678-93.

100. Dolabela F. Teoria Empreendedora dos Sonhos. In: Jardim J, Franco JE, editors. Empreendipédia - Dicionário de Educação para o Empreendedorismo. Lisboa: Gradiva; 2019. p. 713-8.

101. Jardim J, Soares JH, Moutinho A, Calheiros C, Cardoso P, Cardoso MS, et al. Brincadores de Sonhos - Roteiro para Docentes e Formadores. Lisboa: Theya; 2015.

102. Lackéus M, Sävetun C. Assessing the Impact of Enterprise Education in Three Leading Swedish Compulsory Schools. J Small Bus Manag. 2019 Jul 1;57(sup1):33-59.

103. Franco JE. Capital Social. In: Jardim J, Franco JE, editors. Empreendipédia - Dicionário de Educação para o Empreendedorismo. Lisboa: Gradiva; 2019. p. 104-6.

104. Franco JE. Cristianismo e solidariedade: A utopia da misericórdia. Brotéria. 2014;179:45-55.

105. Franco JE, Jardim J. Para um projecto de educação integral Segundo Manuel Antunes, SJ e um novo programa de competências. Linhas. 2008;9(2):24-43.

106. Franco JE. Europa ao Espelho de Portugal: Ideia(s) de Europa na cultura portuguesa. Lisboa: Temas e Debates; 2020.

107. Fiolhais C, Franco JE, Paiva JP, editors. História Global de Portugal. Temas e Debates; 2020.

108. Vega MP. “La fábrica de sueños”: Programa de Educación Emprendedora para alumnos de la escuela primaria y media. Ing Solidar. 2015 Dec 1;11(18):35-9.

109. McCollough MA, Devezer B, Tanner G. An alternative format for the elevator pitch. Int J Entrep Innov. 2016;17(1):5564.

110. Jardim J, Silva H. Estragégias de educação para o empreendedorismo. In: Jardim J, Franco JE, editors. Empreendipédia - Dicionário de Educação para o Empreendedorismo. Lisboa: Gradiva; 2019. p. 338-42. 